\title{
Evaluation of a Method for Revising Colors of Book Cover Images to Reflect Reader Impressions
}

\author{
Tomoko Kajiyama
}

\begin{abstract}
The image on a digital book cover gives potential buyers not only an impression of the book's contents but also serves as an aid when searching and browsing before and after buying the book. I have developed a method for revising book cover images to reflect readers' impressions. I constructed a color database of the correspondence between colors and typical adjectives representing various inner emotional states and a feature database for target products. Using this database and extracting reader impressions from book reviews, I applied appropriate colors to book cover images to reflect reader impressions. I evaluated this method by performing three experiments, one with 42 participants to determine how this method could reflect reader impressions, one with 113 participants to determine how changing colors of book cover image changed the viewer's impression of the book, and one with 7 design experts to determine how this method could help them simulate book cover design. The results revealed that some revised images better reflected the reader impressions than the original images, which might increase sales and give design experts new ideas for the book cover design.
\end{abstract}

Index Terms-Adjective, book cover image, book review, color psychology.

\section{BACKGROUND AND MOTIVATION}

A book cover serves an important function-it gives potential buyers an impression of the book. Digital books sold online [1], [2] that have been in the public domain or are original digital books are usually provided without book cover images. They are often given images containing only the book title with a standard design. The colors in the image are defined on the basis of the genre or are simply random. As a result, the cover image is of little use in searching for a book and browsing the book contents both before purchasing a book and when it is on the purchaser's virtual bookshelf. Several services and applications have been developed for browsing a book's contents. A service has been developed for designing an image for the cover of a digital book [3], and an application has been developed that enables users to design such an image themselves [4]. However, using this service or application is costly or time-consuming.

To support a user's search for a digital book, it is important to create book cover images that reflect the contents. In general, the higher the person's expectations before reading, the lower the level of satisfaction after reading, and the lower

Manuscript received January 7, 2018; revised April 5, 2018. This work was supported in part by a Japan Society for the Promotion of Science Grant-in-Aid for Young Scientists (B) (No. 20454085) and the Telecommunications Advancement Foundation.

Tomoko Kajiyama is with the Open University, Milton Keynes, MK7 6AA, United Kingdom (e-mail: tomoko.kajiyama@open.ac.uk). the expectations before reading, the greater the chances of opportunity loss [5]. It is thus important to reduce the gap between the impression obtained from a book cover image and the impression after reading the book. To realize a function that can do this, we have to consider not only the contents of the book but also the impressions of its readers. Reader impressions can be found in the user reviews commonly found on sites selling digital books. Users can read the reviews and use them to make a purchase decision. However, a user's emotions and latent comments about a book are better represented in images, not text [6]. In addition, the meaning of something can generally be understood more quickly from an image than from words [7].

I have developed a method for automatically revising book cover images so that they reflect reader impressions [8]. I focused on color because studies on color psychology have shown that colors in images are generally the most significant aspect [9]. To develop a color conversion method that extracts color from texts, I focused on the relationships between colors and adjectives because adjectives typically represent one's inner emotional state. I constructed a database showing the correspondence between adjectives and colors and created a method for extracting color from book reviews. To evaluate this method, performing three experiments, one with 42 participants to determine how this method could reflect reader impressions, one with 113 participants to determine how changing colors of book cover image changed the viewer's impression of the book, and one with 7 design experts to determine how this method could help them simulate book cover design.

\section{OVERVIEW OF THE PROPOSED METHOD}

The impressions and feelings expressed by readers in their book reviews are visualized by extracting the words describing them. Colors are identified by extracting adjectives because emotion that represents an inner state is described using adjectives [10]. Fig. 1 shows the flow of this proposed method. The color database was constructed using a color image scale [11] expressing the relationships between adjectives and colors. This scale defines 180 adjectives representing basic emotions and 130 colors that capture experiences psychologically for Japanese people. The database has three attributes: color (RGB), adjective, and frequency of use. Each color corresponds to more than 1 but less than 25 adjectives. The 180 adjectives were extended to 3184 by using a thesaurus [12].

A book image that reflects reader impressions is created by replacing feature colors in the original image with the colors extracted from the book reviews. The procedure is as follows. 
(1) Adjectives are extracted by analyzing the morphology of the input book reviews.

(2) For each book review, scores for colors are calculated on the basis of the total number of occurrences of each adjective and the relationships between adjectives and colors as expressed in a color database [13].

(3) Scores for colors for all reviews are calculated by counting up the normalized scores for colors for each review.

(4) An original image is converted using the colors included in the color database by selecting the nearest color in the RGB color space.

(5) Two areas, $A_{1}$ (the area with the most frequent color) and A2(the area with the second most frequent color), are selected from the converted image.

(6) Area $A_{1}$ is converted into the color with the highest score calculated in step (3).

(7) The most frequent color among the colors adjacent to area Azselected in step (5) is selected.

(8) The distances in the $\mathrm{L}^{*} \mathrm{a} * \mathrm{~b}$ color space [14] between the color selected in step (7) and the colors with high scores calculated in step (3) are calculated.

(9) Area $A_{2}$ is converted into the color which has a long distance $(>25)$ calculated in step (8) with the highest score calculated in step (3) excluding the color used in step (6).

Pre-testing for analyzing the original book cover images was performed for books in the category related to novels on a book shopping site. The books which got a high sales ranking were crawled except for the books having similar design patterns depending on the genre or author. The original book cover images were also crawled and resized to have the same width. For over half the books, the color of area $A 1$ was a background color, and the color of area $A_{2}$ was related to descriptive information such as title and author. The colors adjacent to area $A$ 2 were considered to maintain the visibility of this information [15]. Table I shows examples of revised images and the original and revised colors.

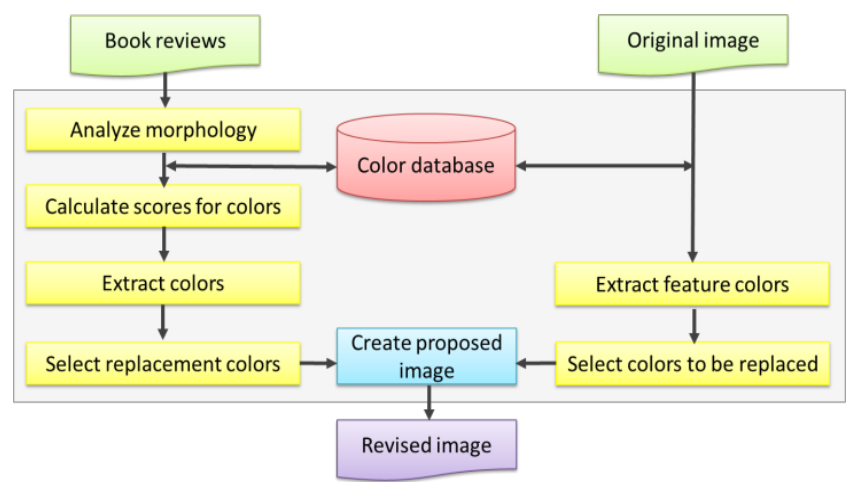

Fig 1. Flow of proposed method.

TABLE I: EXAMPLES OF REVISED IMAGES

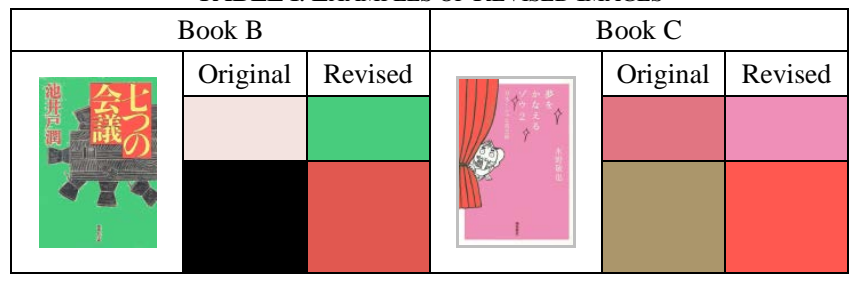

\section{Evaluation METHOD}

\section{A. Objective and Preparation}

To evaluate the effectiveness of this method, I performed three experiments with different objectives: (a) how does revising the book cover image reflect the reader impressions, (b) how does it change the desire to read, and (c) how does it help book cover designers. Six books (novels), each with over 175 book reviews, were selected from a book shopping site on the basis of differences in design, areas of the book cover to be modified and book category (including the two books shown in Table I) as revealed by the results of the pre-testing. Each book got a high sales ranking in each book category in an online book shopping site (Amazon.co.jp.) All participants of all experiments were Japanese because the perception of colors depends on the culture of the beholder and the color database using this method was constructed based on Japanese people. Table II shows the features for books used in all experiments. The score ranking indicates the score calculated in step (3) described in Section 2.

TABLE II: BOOKS USED IN ALL EXPERIMENTS

\begin{tabular}{|c|c|c|c|c|c|}
\hline book & category & $\begin{array}{l}\text { no. of } \\
\text { reviews }\end{array}$ & revised area & $\begin{array}{c}\text { \% of } \\
\text { pixels } \\
\text { converted }\end{array}$ & $\begin{array}{c}\text { score } \\
\text { ranking }\end{array}$ \\
\hline \multirow{2}{*}{ A } & \multirow{2}{*}{$\begin{array}{l}\text { life, health, } \\
\text { child raising }\end{array}$} & \multirow{2}{*}{175} & background & 64.36 & 1 \\
\hline & & & background & 6.92 & 2 \\
\hline \multirow[b]{2}{*}{ B } & \multirow{2}{*}{$\begin{array}{l}\text { economy, } \\
\text { social }\end{array}$} & \multirow[b]{2}{*}{314} & background & 43.21 & 1 \\
\hline & & & $\begin{array}{l}\text { title palate \& } \\
\text { author name }\end{array}$ & 11.03 & 3 \\
\hline \multirow{2}{*}{$\mathrm{C}$} & \multirow{2}{*}{$\begin{array}{l}\text { hobby, self- } \\
\text { development }\end{array}$} & \multirow{2}{*}{305} & background & 77.33 & 1 \\
\hline & & & object (curtain) & 16.21 & 2 \\
\hline \multirow{2}{*}{$\mathrm{D}$} & \multirow{2}{*}{$\begin{array}{c}\text { art and } \\
\text { literature }\end{array}$} & \multirow{2}{*}{247} & background & 44.70 & 1 \\
\hline & & & background & 19.98 & 3 \\
\hline \multirow{2}{*}{$\mathrm{E}$} & \multirow{2}{*}{$\begin{array}{l}\text { SF, horror, } \\
\text { fantasy }\end{array}$} & \multirow{2}{*}{286} & background & 34.82 & 1 \\
\hline & & & object (brain) & 8.93 & 2 \\
\hline \multirow{2}{*}{$\mathrm{F}$} & \multirow{2}{*}{$\begin{array}{l}\text { mystery, } \\
\text { suspense }\end{array}$} & \multirow{2}{*}{179} & background & 50.46 & 1 \\
\hline & & & title plate & 13.21 & 2 \\
\hline
\end{tabular}

\section{B. Procedure}

In the first experiment to determine objective (a), all of participants ranged in age from 20 to 54 . They read their assigned novels over three months ago and remembered the story, however, they forgot the design of the original book cover image. They gave their impressions of both images. A seven-point scale (from 1 to 7) was used to answer the questions, and the participants explained the reason for each score. The larger the number, the more they thought that the image reflected their impression; a score of 4 represented a neutral impression. Because some of them evaluated the image for more than one book, 30 participants were assigned for each book. The order of the books was random for each participant who was assigned several books.

In the second experiment to determine objective (b), all participants ranged in age from 20 to 55. None had read their assigned novels, however, all of them like reading books especially in the same categories of the assigned novels. They were divided into two groups: the original book cover image was displayed in one group and the revised image was displayed in the other group. After displaying the assigned image with book information including abstract and reader 
reviews, participants gave the degree of desire to read the book. A seven-point scale (from 1 to 7 ) was used to answer the questions, and the participants explained the reason for each score. The larger the number, the more they thought that they wanted to read the book; a score of 4 represented a neutral impression. Because some of them evaluated the image for more than one book, 60 participants were assigned for each book. The order of the books was random for each participant who was assigned several books.

In the third experiment to determine objective (c), there were 7 participants who were design experts, ranging in age from 32 to 56 . The features of the participants are shown in Table III. Note that the female participants had fewer years of experience than the male participants. All participants knew or had read the target books. I displayed the original image and revised image and asked the participants which one was more appropriate as the book cover image. I also asked the reason for the selection. This procedure was repeated for each of the target books. The book order was random for each participant.

TABLE III: FEATURES OF PARTICIPANTS

\begin{tabular}{|c|c|c|c|}
\hline $\begin{array}{c}\text { Partic- } \\
\text { ipant }\end{array}$ & Gender & $\begin{array}{c}\text { Years as } \\
\text { designer }\end{array}$ & Design experience \\
\hline \hline 1 & female & 6 & software \\
\hline 2 & female & 9 & product package \\
\hline 3 & female & 10 & product package \\
\hline 4 & male & 21 & food package, book cover \\
\hline 5 & male & 25 & product package \\
\hline 6 & male & 34 & $\begin{array}{c}\text { food package, product package, } \\
\text { book cover, software }\end{array}$ \\
\hline 7 & male & 40 & $\begin{array}{c}\text { food package, product package, } \\
\text { book cover }\end{array}$ \\
\hline
\end{tabular}

\section{RESUlTS AND DisCUSSION}

\section{A. Reflecting Reader Impressions}

To estimate the reduction in the gap between the impression after reading and the impressions upon viewing the book cover image, I compared the average scores for the original book cover image and the revised image. Table IV shows the results of t-test. For the target books taken as a whole, the average score of the impression upon viewing the revised image was higher than that of the impression upon viewing the original image, though the difference was not statistically significant as shown in the last line of Table IV.

However, there were significant differences in four books when analyzing each book individually. The average scores of the impression upon viewing the revised images were higher than that of the impression upon viewing the original image for three books (book A, book C and book E) except for book B. Book A could reflect reader impressions such as feeling happy, feeling cheerful, and heart-warmed by changing a bluish color to a pinkish color and a whitish color to a yellowish color. Book E could reflect reader impressions such as silent, nonplused and mild by changing reddish colors to a greenish color and orange. For book C, as show in Table II, the original image was changed to two similar pinkish colors. These colors could reflect reader impressions such as feeling joyful, open-minded and peaceful.

On the other hand, book B could not reflect reader impressions such as positive, auspicious and courageous. It is considered that there were two reasons were proposed why the average scores of the revised image were lower: the type of novel and the arrangement of colors. The number of reviews was the biggest of the target books, however the number of extracted adjectives in step (1) described in Section 2 and their occurrences were fewer because this novel described one social problem. Although the reader impressions were weak in the first place, over half the area of the book cover image was modified by these two colors. This means that the extent of the area manipulated need to be considered based on the features of extracted adjectives from reader reviews. Many participants who gave a lower score for the revised image said that the revised image was too garish because the combination of colors was complex and they were confused when examining the contents of the book. I focused on visibility to select and convert colors in this method, however, this means that multiple colors need to be considered in conjunction with the original colors to reflect multiple impressions.

The average scores of the revised images for the two books which did not have significant differences (Book D and Book F) were slightly lower than those of the original images. Based on the participants' answers, it is considered that the reason stems from the modified colors of the book cover blending with a part of the object used to partly represent the contents of the book. For book D, black and a brownish color were changed to bluish and purplish colors. Because the main character of this story is a piano tuner, a part of the piano was drawn in black and a music stand was drawn in a brownish color abstractly. For book F, the scene of consoling the spirit of deceased people in the river at night was drawn. The river at night drawn in black was changed to a purplish color by this method. This means that the original colors of the objects need to be considered when changing other colors. In these two books, the object colors were used as background and there were few areas which could be changed. A different way of reflecting reader impressions to the original book cover image, e.g., creating new image based on the original image with frame in color expressing reader impressions, might need to be considered.

TABLE IV: RESULTS OF T-TESTS

\begin{tabular}{|c|c|c|c|c|c|}
\hline \multirow{2}{*}{ book } & \multicolumn{2}{|c|}{ average score } & $\begin{array}{c}\text { degrees of } \\
\text { freedom }\end{array}$ & \multirow{2}{*}{-value } & $p$-value \\
\cline { 2 - 4 } & original & revised & 29 & 9.204 & $p<.001^{* *}$ \\
\hline A & 4.167 & 5.367 & 29 & 8.097 & $p<.001^{* *}$ \\
\hline B & 6.067 & 4.333 & 29 & 8.102 & $p<.001^{* *}$ \\
\hline C & 3.900 & 5.600 & 29 & 0.769 & 0.224 \\
\hline D & 4.033 & 3.933 & 29 & 1.963 & $0.030^{* *}$ \\
\hline E & 4.000 & 4.300 & 29 & 1.361 & 0.092 \\
\hline F & 5.133 & 4.833 & 179 & 1.612 & 0.054 \\
\hline all & 4.550 & 4.727 & & ${ }^{*} p<0.05,{ }^{* *} p<0.01$ \\
\hline
\end{tabular}

\section{B. Desire to Read}

It is important that the revised book cover image does not lead to a reduction in sales even though it reflects reader impressions. To check this factor, I compared the desire to read between viewing the original image and viewing the revised image. Table $\mathrm{V}$ shows the results for the average scores for both images and the results of t-tests. For all of the 
target books, the average score of the impression upon viewing the revised image was higher than the ones of the impression upon viewing the original image, though the difference was not statistically significant as shown in the last line of Table $\mathrm{V}$.

However, there were significant differences in four books when analyzing each book individually. For three books which confirmed that reader impressions were reflected, there were significant differences for book $\mathrm{C}$ and book $\mathrm{E}$. The average score of the revised image for book A was higher than that of the original image, however, the difference was not statistically significant. Considering the participants' comments, this result depended on the target of this book, that is, mothers especially those who have daughters. A $t$-test for only female participants showed that there was a significant difference $(t(36)=1.980, p<0.05)$. The results revealed that the revised images better reflected the reader impressions than the original images and therefore might increase sales.

The result for book $\mathrm{B}$ was the same result as the first experiment and the revised image did not reflect reader impressions. According to the participants' comments, some of them wanted to read the book when reading user reviews, however, they gave up because they felt the gaps between the contents of the book and the impressions upon viewing the revised image were too large. The result for book $\mathrm{F}$ was the same result as the first experiment and no differences were statistically significant.

On the other hand, regarding the result for book $\mathrm{D}$, the average score of the revised image was higher and there was a significant difference. In the participants' comments, there were no words related to the piano and they described words related to life and growth. The modified colors were expressing impressions such as feeling successful, sophisticated and romantic. It was considered that they did not notice a part of the piano on the original book cover image and they evaluated it with only colors modified in this method.

TABLE V: RESULTS OF T-TESTS

\begin{tabular}{|c|c|c|c|c|c|}
\hline \multirow{2}{*}{ book } & \multicolumn{2}{|c|}{ average score } & degrees of & \multirow{2}{*}{-value } & $p$-value \\
\cline { 2 - 3 } & original & revised & freedom & & \\
\hline A & 4.066 & 4.400 & 45 & 0.751 & 0.228 \\
\hline B & 5.600 & 4.566 & 58 & 5.040 & $p<.001^{* *}$ \\
\hline C & 4.333 & 5.467 & 58 & 5.277 & $p<.001^{* *}$ \\
\hline D & 5.233 & 5.733 & 58 & 2.126 & $0.019^{*}$ \\
\hline E & 4.100 & 4.533 & 58 & 1.705 & $0.047^{*}$ \\
\hline F & 4.667 & 4.233 & 58 & 1.411 & 0.082 \\
\hline all & 4.667 & 4.877 & 179 & 1.610 & 0.055 \\
\hline
\end{tabular}

\section{Helping to Design Book Covers}

For book A, four participants excluding participants 4 and 5 , and selected the revised image as the one more appropriate for the book cover. For book C, five participants excluding participants 3 and 7 selected the revised image as the one more appropriate for the book cover. For these two books, the reasons they selected it were related to the impression extracted from reader reviews. For example, for book A, they selected it based on the impression of "happy" or "heartwarming”, e.g., "the pinkish colors give an impression of a brilliant life," and "the majority of the book cover is drawn with the colors women like."

For the other four books, two participants or fewer selected the revised images as the one more appropriate for the book cover. The reasons for selected the original images were related to harmony of colors and visibility of text. For book B, none selected the revised image. According to the designers' comments, four colors including two modified colors were too complex to give the impression of the contents of the book to potential readers. In fact, the original image was designed with monotone colors except for one beige color. Because this issue was pointed out in the first experiment, it is vital future work for multiple colors to be considered in conjunction with the original colors to reflect multiple impressions. For book D, one participant selected the revised image as the one more appropriate for the book cover. According to the designers' comments, the text such as title, author and publisher did not stand out. This means that more consideration of visibility is needed when selecting the replacement colors.

For book E, in the first and second experiments, there were significant differences between the scores for the original image and the scores for the revised image. However, only two participants selected the revised image as the one more appropriate for the book cover. The reason for selecting the original image was the same reason for book B. This indicates that there might be differences in impressions between ordinary people and design experts, meaning that revising images using my proposed method may in fact give design experts a better understanding of which book cover designs are more suitable for ordinary people.

For book F, two participants selected the revised image as the one more appropriate for the book cover. The reason for selecting the original image was the same reason for book B. However, the reason for selecting the revised image was related to the unexpected design. For example, "the overall design was mysterious as there seems to be no harmony in the choice of colors" and "the design balance is really unique although there is a tendency to hesitate using a greenish color to represent the notion of 'passionate'”. The results indicate that revising images using my proposed method may give design experts new ideas while simulating book cover design. In addition, one of them suggested that the application of this method may help non-experts select appropriate colors. The additional experimentation for design tasks with non-expert designers may offer better results than this.

\section{CONCLUSION}

I have developed a method for automatically revising book cover images so that they reflect reader impressions. I evaluated this method experimentally by having 42 participants give their impressions, having 113 participants rate their desire to read and having 7 design experts select the more appropriate book cover image from the original image and the revised image.

I found that (1) displaying appropriately revised images can give the impressions that were closer to the impression after reading than original images, (2) the revised images may increase sales, and (3) displaying an appropriately revised image may give design experts new ideas for book cover 
design. To create an effective book cover image better reflecting reader impressions, it is necessary to improve the algorithm for analysis in conjunction with the original colors to reflect multiple impressions, to improve the algorithm for maintaining a good balance between the contents of the book and reader impressions, and to improve the algorithm so that visibility is considered when selecting the replacement colors.

\section{ACKNOWLEDGMENT}

I am grateful to Prof. Stefan Rüger for useful discussions and advice. I would like to thank the members of the Knowledge Media Institute of The Open University for their hospitality during my visit, when the main results of this paper were obtained. I also like to take this opportunity to thank all of the participants for the experiments.

\section{REFERENCES}

[1] Aozora Bunko. [Online]. Available: http://www.aozora.gr.jp/

[2] Calibre. [Online]. Available: http://calibre-ebook.com/

[3] Libura. [Online]. Available: http://libura.com/

[4] Interwired Timely Research. [Online]. Available: http://www.dims.ne.jp/timelyresearch/2009/090202/

[5] R. M. Dawes, D. Singer, and F. Lemons, "An experimental analysis of the contrast effect and its implications for intergroup communication and the indirect assessment of attitude," Journal of Personality and Social Psychology, vol. 21, no. 3, pp. 281-295, 1972.

[6] N. Akamatsu, "The effects of first language orthographic feature on second language reading in text,” Language Learning, vol. 53, no. 2 , pp. 207-231, 2003.

[7] K. Shimazaki, Psychological Color, Japan: Bunka-shobo-hakubunnsha, 1990.
[8] T. Kajiyama, "Color conversion of book cover images to reflect reader impressions”, in Proc. 2016 IEEE 5th Global Conference on Consumer Electronics, 2016, pp.608-609.

[9] T. Oyama, Introduction of Color Psychology, Japan: Chuokoron-shinsha, 1994.

[10] M. Ohso, "Verbs and adjectives of emotion in Japanese," Studies in Language and Culture, vol. 22, no. 2, pp. 21-30, 2001.

[11] S. Kobayashi, Color Image Scale, Japan: Kodansha, 2001.

[12] Weblio Thesaurus. [Online]. Available: http://thesaurus.weblio.jp/

[13] T. Iida, T. Kajiyama, N. Ouchi, and I. Echizen, “A color extraction method from text for use in creating a book cover image that reflects reader impressions,” Advances in Intelligent Systems and Computing, Springer, vol. 264, pp. 201-211, 2016.

[14] Color space transformation. [Online]. Available: http://w3.kcua.ac.jp/ fujiwara/infosci/colorspace/colorspace3.html

[15] Nippon Denshoku Industries. Information of colors and lights. [Online].

Available: http://www.nippondenshoku.co.jp/web/english/colorstory/08_allowan ce_by_color.htm

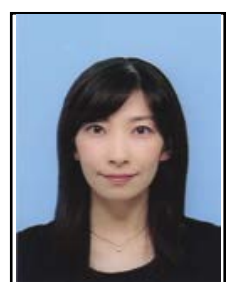

Tomoko Kajiyama received a B.Sc. from Tsuda College in 2001, an M.E. from Keio University in 2004, and a Ph.D. from the Graduate University for Advanced Studies at the National Institute of Informatics in 2007.

She worked as a research associate in the School of Human Sciences at Waseda University from 2007 to 2010. She became a research associate in the College of Science and Engineering at Aoyama Gakuin University in 2010 and an assistant professor in 2013. She became a visiting researcher in Knowledge Media Institute at the Open University in 2017.

Her research interests include search user interfaces, multimedia databases, and knowledge interaction design. Her paper about the vision of the future for multimedia environment received the best paper award from ITE (the Institute of Image Information and Television Engineers). 